\title{
Comparing Watchful Waiting Approach vs. Antibiotic Therapy in Children with Nonsevere Acute Otitis Media: A Randomized Clinical Trial
}

\author{
Leila Shahbaznejad $\left(\mathbb{D},{ }^{1}\right.$ Ensieh Talaei $(\mathbb{D}),{ }^{1}$ Fatemeh Hosseinzadeh $\left(\mathbb{D},{ }^{1}\right.$ Baraneh Masoumi $\left(\mathbb{D},{ }^{1}\right.$ \\ Shaghayegh Rezai ${ }^{D},{ }^{2}$ and Mohammad Sadegh Rezai ${ }^{1}{ }^{1}$
}

${ }^{1}$ Pediatric Infectious Diseases Research Center, Communicable Diseases Institute, Mazandaran University of Medical Sciences, Sari, Iran

${ }^{2}$ Department of Microbiology and Virology, Mashhad University of Medical Sciences, Mashhad, Iran

Correspondence should be addressed to Mohammad Sadegh Rezai; drmsrezaii@yahoo.com

Received 22 February 2021; Revised 28 April 2021; Accepted 11 May 2021; Published 28 May 2021

Academic Editor: Asirvatham Alwin Robert

Copyright ( $) 2021$ Leila Shahbaznejad et al. This is an open access article distributed under the Creative Commons Attribution License, which permits unrestricted use, distribution, and reproduction in any medium, provided the original work is properly cited.

\begin{abstract}
Objective. To compare both approaches for the treatment of nonsevere acute otitis media (AOM) in Iran. Methods. This randomized clinical trial was performed at a pediatric infectious diseases clinic in Buali tertiary hospital in Sari, north of Iran, from 2016 to 2018. All participants in this study were previously healthy children with AOM diagnosis, who were 6 months to 6 years old. The patients were randomly assigned into two groups: the intervention $(80 \mathrm{mg} / \mathrm{kg} / \mathrm{day}$ amoxicillin for 7-10 days) and the control group (watchful waiting approach). AOM recovery and adverse drug reactions were evaluated after 72 hours, and the patients were followed for the frequency of AOM and middle ear effusion 1 and 3 months' postintervention. Results. A total of 396 children have participated in this study. AOM recovery was significantly different in the two groups ( $73 \%$ vs. $44 \%$ in the intervention and control groups, respectively). Recurrence of AOM and middle ear effusion (MEE) persistence, one month following the intervention, have not shown any significant differences between the two groups. However, the AOM recurrence between 1 and 3 months was more frequent in the control group. The frequency of diarrhea was also higher in the intervention group compared to the control but no significant difference was found between the two groups regarding vomiting and skin rash. Conclusion. The faster recovery from AOM is achieved when an antibiotic treatment regimen is applied, although the risk of potential side effects should be considered.
\end{abstract}

\section{Introduction}

Acute otitis media (AOM) is one of the most common childhood diseases. Nearly $75 \%$ of children experience at least one episode of ear infection before starting their school $[1,2]$. $\mathrm{AOM}$ is one of the leading causes of healthcare visits in many countries. The worldwide AOM rate is estimated to be about $10.85 \%$ (709 million cases) each year, from which $51 \%$ of them are under the age of five [3].

Age, gender, race, genetics, socioeconomic status, neonatal feeding, smoke exposure, day care attendance, season, and the midline facial defects are the most common factors affecting the rate of $A O M$ infections in children $[1,3,4]$. Otitis media is more common in boys aged 6 to 20 months [5].
Socioeconomic status can result in many risk factors associated with AOM. Children from lower socioeconomic status may be at more risk of smoke exposure, crowded day care centers, poorer living condition, bottle-feeding, and more viruses and bacterial pathogens [6].

Otitis media more frequently occurs during the cold seasons, due to the increased number of upper respiratory tract infections [7]. Viruses are the most common cause of AOM infections. In addition, some bacteria can cause $\mathrm{AOM}$ as well such as Streptococcus pneumonia, nontypeable Haemophilus influenza, and Moraxella catarrhalis. Other pathogens that are less frequently associated with AOM are Streptococcus type A, staphylococcus aureus, and Gram-negative organisms which are more commonly seen in neonates $[8,9]$. 
Currently, most guidelines suggest two methods of treatment for AOM [10]. The treatment options consist of the use of antibiotics and the "watchful waiting" approach, which was first suggested by the American Academy of Pediatrics (AAP) and American Academy of Family Physicians (AAFP) in 2004. The watchful waiting approach was introduced due to the emergence of antibiotic resistance. The guideline was then modified in 2013 with more emphasis on the importance of accurate diagnosis of AOM [11]. According to the recent guideline, the watchful waiting approach can be used in healthy children with 6-23 months of age who have mild symptoms with appropriately diagnosed unilateral AOM or children $\geq 2$ years old with bilateral or unilateral AOM or children who do not fully meet the diagnostic criteria [12]. The watchful waiting approach can be used for 48 hours if the follow-up is assured. Children $\geq 6$ months with a bulging tympanic membrane, fever $\left(\geq 39^{\circ} \mathrm{C}\right)$ and moderate to severe systemic illness, who have severe otalgia, or have already been significantly ill for $\geq 48$ hours and children $<2$ years with bilateral AOM regardless of the additional signs or symptoms should be treated with antimicrobial agents [12, 13]. Studies show that up to $80 \%$ of AOM cases resolve spontaneously without antibiotics, and antibiotics may increase the risk of vomiting, diarrhea, and rash and also antibiotic resistance $[10,14]$.

Results of a study in Sweden showed that pneumococcal conjugate vaccines reduce the incidence of otitis media up to $26 \%$ and delay the first episode of otitis media in infants and young children [15]. This could be due to the reduced nasopharyngeal colonization of bacteria by PCVs [16]. In 2010, the FDA approved the 13-valent pneumococcalconjugated vaccines (PCV13), which has shown to reduce the prevalence of AOM in children under 2 years old. This vaccine has been included in their national vaccination program since [4]. However, pneumococcal vaccination is not still included in the national vaccination program of some countries [17].

Neither conjugated and polysaccharide pneumococcal vaccines nor Hib vaccine are routine as part of the national immunization program in Iran. So, in this study, we aimed to compare the success rate of treatment options, potential side effects, and recurrence of AOM in the observational and antimicrobial groups in Iranian children with AOM, who have not received pneumococcal vaccines as part of their national vaccination program.

\section{Methodology}

2.1. Study Design and Interventions. This randomized clinical trial was undertaken in the pediatric infectious diseases clinic at Buali tertiary hospital in Sari, north of Iran, between 2016 and 2018. The eligibility criteria for this study included age (6 months to 6 years), AOM diagnosis (acute onset of fever, erythema of tympanic membrane, and middle ear effusion), and onset of the symptoms within 48 hours prior to visiting the physician. Children with severe AOM (fever $\geq 39^{\circ} \mathrm{C}$, moderate to severe irritability, and otalgia), otorrhea, concurrent conjunctivitis, underlying diseases such as immunodefi- ciency, and history of penicillin allergy were excluded from the study.

According to the AAP guideline 2013, patients included in the inclusion criteria were randomly assigned into two groups, the intervention group and the control group [11].

2.2. Intervention. The intervention group received a high dose $(80 \mathrm{mg} / \mathrm{kg} /$ day) of amoxicillin suspension made by Farabi pharmaceutics with the brand name of "Faramox BD" for 7-10 days divided into two doses. For the control group, the "watchful waiting" approach and monitoring were performed. In case of pain and fever, acetaminophen or ibuprophen was prescribed for patients of both groups.

2.3. Measures. Clinical symptoms and adverse drug reactions including fever, otalgia, irritability, poor feeding, seizure, vomiting, diarrhea, coryza, cough, pharyngitis, nasal congestion, and allergic reactions were recorded in both groups at the first and fourth days of intervention. Severe complications of AOM such as mastoiditis, petrositis, meningitis, and otorrhea were also evaluated during the study. AOM recovery was defined as fever, irritability, and otalgia elimination after 72 hours.

The outcome measures included recurrent AOM and middle ear effusion (MEE) after one and three months following the intervention. MEE was defined as otorrhea, loss of tympanic membrane mobility, air-fluid level or bubbles behind tympanic membrane, and tympanic edema. AOM was defined as fever in addition to middle ear inflammation and effusion.

2.4. Randomization and Mmasking. The patients were randomly divided into two groups, the intervention and control groups, by simple randomization method, and physicians were given a table of precoded numbers and patients enrolled the study in order of table numbers. The total sample size consisted of 400 patients, 200 participants per group. Neither the participants nor the evaluators were aware of the randomization process or group allocation. After obtaining the written informed consent, amoxicillin suspension was given to the parents of the intervention group. So, this study was not blinded.

2.5. Ethical Considerations. The ethics committee of Mazandaran University of Medical Sciences approved the study protocol (Code: IR.MAZUMS.REC.1395.2489), and it was registered in the Iranian Registry of Clinical Trials (Code: IRCT20111224008507N2). A written informed consent was obtained from all parents of children prior to enrolment. The trial was conducted in accordance with the principles of the Declaration of Helsinki.

2.6. Statistical Analyses. The collected data were analyzed using the 2011 Statistical Package for the Social Sciences (SPSS; IBM, Armonk, New York) software for Windows, version 20. Chi-square, Fisher's exact, and McNemar tests were used to compare the variables before and after the intervention, and the $P$ value of less than 0.05 was considered being statistically significant. 


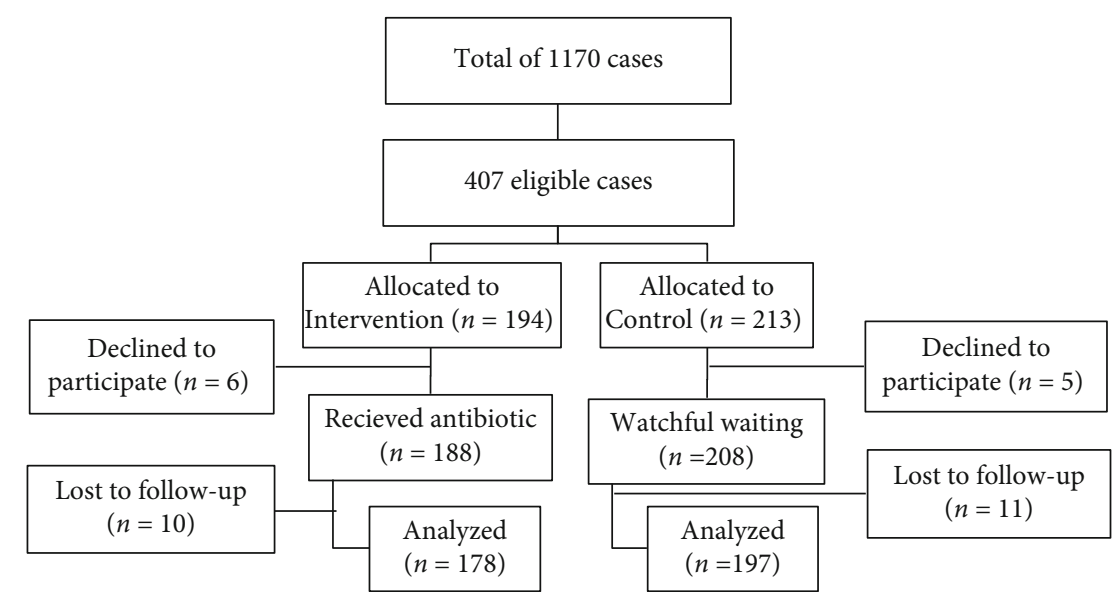

Figure 1: Flow chart of the study allocation and follow-up.

Table 1: Demographic and characteristics of the patients.

\begin{tabular}{|c|c|c|c|c|}
\hline & & & & $P$ yalue \\
\hline & & Intervention $(N: 188)$ & Control (N: 208) & $P$ value \\
\hline Condor & Male & $104(55)$ & $95(46)$ & $>005$ \\
\hline Gender & Female & $84(45)$ & $113(54)$ & $>0.05$ \\
\hline Ano oroun (months) & $6-23$ & $92(49)$ & $97(47)$ & $>0.05$ \\
\hline Age group (months) & $>24$ & $96(51)$ & $111(53)$ & $>0.05$ \\
\hline Voccinotion $5 \mathrm{C}$ & Yes & $188(100)$ & $208(100)$ & $>005$ \\
\hline Vaccination & No & $0(0)$ & $0(0)$ & $>0.05$ \\
\hline Dpoumercol uccinotion & Yes & $3(1.6)$ & $3(1.4)$ & \\
\hline Pneumococcal vaccination & No & $185(98.4)$ & $205(98.6)$ & $>0.05$ \\
\hline Antibintinin lot 1 und & Yes & $109(58)$ & $136(65)$ & 0005 \\
\hline Antibiotic in last 4 weeks & No & $79(42)$ & $72(35)$ & $>0.05$ \\
\hline & Exclusive breastfeeding & $128(68)$ & $136(66)$ & \\
\hline History of breastfeeding & Formula-fed & $24(13)$ & $28(14)$ & $>0.05$ \\
\hline & Both & $36(19)$ & $41(20)$ & \\
\hline & Day care center & $48(26)$ & $57(27)$ & \\
\hline Child care type & Babysitter & $10(5)$ & $8(4)$ & $>0.05$ \\
\hline & Mother & $130(69)$ & $143(69)$ & \\
\hline 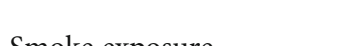 & Yes & $30(16)$ & $37(18)$ & 0005 \\
\hline smoke exposure & No & $156(84)$ & $169(82)$ & $>0.05$ \\
\hline
\end{tabular}

\section{Results}

A total of 407 children were enrolled in the study (Figure 1). From these patients, 11 cases ( 6 patients from the case group and 5 from the control) withdrew due to reluctance. 396 children (188 patients in the intervention group, mean age: $29.05 \pm 16.6$ months, and 208 ones in control group, mean age: $28.88 \pm 15.9$ months: $P>0.05)$ have participated in this study, from which 189 children (47.82\%) aged 6-23 months and the rest aged 2-6 years.

No significant differences were found between the two groups regarding the symptoms, clinical findings, types of care, feeding, smoke exposure, antibiotic consumption in the last 4 weeks, and vaccination history (Table 1 ). At the baseline, the most common symptoms or clinical findings in all participants were fever (342 cases, $86.36 \%$ ), otalgia (200 cases, $50.50 \%$ ), and irritability (172 cases, $43.43 \%)$. Frequencies of the unilateral or bilateral erythema and/or effusion were not significantly different in the two groups $(P>0.05)$.

Three days after the intervention, most of the complaints disappeared in both groups, but the improvements were more significant in the antibiotic group. 28 (15\%) patients from the intervention group and 59 (28\%) patients from the control group still had otalgia $(P<0.01) .35(19 \%)$ of the interventions and 92(44\%) of the controls had a fever $(P<0.01)$, and $19(10 \%)$ of the interventions and $40(19 \%)$ of the controls had irritability $(P<0.05)$ (Table 2$)$. Overall, AOM recovery was seen in $137(73 \%)$ and 91 (44\%) of the 
TABLE 2: Clinical symptoms of the patients before and after intervention.

\begin{tabular}{|c|c|c|c|c|c|c|}
\hline \multirow[b]{2}{*}{ Variables } & \multicolumn{3}{|c|}{ Before intervention } & \multicolumn{3}{|c|}{ After intervention } \\
\hline & Intervention ( $N: 188)$ & Control (N: 208) & $P$ value & Intervention $(N: 188)$ & Control (N: 208) & $P$ value \\
\hline Otalgia & $94(50 \%)$ & $106(51 \%)$ & $>0.05$ & $28(15 \%)$ & $59(28 \%)$ & $<0.01^{*}$ \\
\hline Fever & $170(90 \%)$ & $172(83 \%)$ & $<0.01^{*}$ & $35(19 \%)$ & $92(44 \%)$ & $<0.01^{*}$ \\
\hline Irritability & $86(46 \%)$ & $86(41 \%)$ & $>0.05$ & $19(10 \%)$ & $40(19 \%)$ & $<0.05^{*}$ \\
\hline Vomiting & $6(3 \%)$ & $9(4 \%)$ & $>0.05$ & $9(5 \%)$ & $4(2 \%)$ & $>0.05$ \\
\hline Diarrhea & $10(5 \%)$ & $8(4 \%)$ & $>0.05$ & $13(7 \%)$ & $5(2 \%)$ & $<0.05^{*}$ \\
\hline Coryza & $107(57 \%)$ & $118(57 \%)$ & $>0.05$ & $6(3 \%)$ & $10(5 \%)$ & $>0.05$ \\
\hline Cough & $92(49 \%)$ & $106(51 \%)$ & $>0.05$ & $4(2 \%)$ & $10(5 \%)$ & $>0.05$ \\
\hline Pharyngitis & $4(2 \%)$ & $1(0.5 \%)$ & $>0.05$ & $0(0 \%)$ & $0(0 \%)$ & - \\
\hline Nasal congestion & $15(8 \%)$ & $10(5 \%)$ & $>0.05$ & $1(0.5 \%)$ & $0(0 \%)$ & $>0.05$ \\
\hline Skin rash & $21(11 \%)$ & $25(12 \%)$ & $>0.05$ & $2(1 \%)$ & $0(0 \%)$ & $>0.05$ \\
\hline Asthma & $7(4 \%)$ & $10(5 \%)$ & $>0.05$ & $0(0 \%)$ & $0(0 \%)$ & - \\
\hline Poor feeding & $4(2 \%)$ & $1(0.5 \%)$ & $>0.05$ & $0(0 \%)$ & $0(0 \%)$ & - \\
\hline Ear drum perforation & $\mathrm{x}$ & $\mathrm{x}$ & & $2(1 \%)$ & $1(0.5 \%)$ & $>0.05$ \\
\hline Unilateral erythema of TM & $104(55 \%)$ & $129(62 \%)$ & $>0.05$ & $* *$ & $* *$ & \\
\hline Bilateral erythema of TM & $83(44 \%)$ & $78(37 \%)$ & $>0.05$ & $* *$ & $* *$ & \\
\hline Unilateral MEE & $93(49 \%)$ & $111(53 \%)$ & $>0.05$ & $* *$ & $* *$ & \\
\hline Bilateral MEE & $55(29 \%)$ & $59(28 \%)$ & $>0.05$ & $* *$ & $* *$ & \\
\hline
\end{tabular}

MEE: middle ear effusion; TM: tympanic membrane. ${ }^{*}$ Statistically significant. ${ }^{* *}$ Not assessed after 3 days. X: ear drum perforation cases were excluded at initiation of the study.

patients from the antibiotic-treated group and the control group, respectively $(P<0.01)$.

Furthermore, a significant patient improvement from AOM was observed in those with unilateral or bilateral erythema of tympanic membrane and/or middle ear effusion in children younger or older than 2 years old regardless of their exposure to antibiotic therapy 4 weeks prior to study participation. Table 3 shows other factors attributed to the improvement of AOM in detail.

Severe complications due to the AOM infections such as meningitis, acute mastoiditis, and petrositis were not seen, but eardrum perforation was reported in two patients from the intervention group and one from the control group $(P>0.05)$ (Table 2).

From a total of 396 patients, 375 cases were followed 1 and 3 months after the intervention. The parents were asked about the recurrence of AOM and MEE symptoms. At the one-month follow-up, no differences were found between the two groups in terms of AOM recurrences. However, observation from the first and three-month follow-up showed lower AOM episodes in the antibiotic-treated group $(P<0.05)$ (Table 4$)$. There was no difference in the recurrence of AOM in both groups based on the history of allergy, asthma, breastfeeding, child care type, smoke exposure, and erythema of tympanic membrane or MEE at the beginning of the study (Table 5). MEE frequency decreased during the time, and it was not significantly different in any of the groups (Table 4).

Drug side effects occurred in 20 (11\%) of the intervention and $6(3 \%)$ of the control group patients (Table 4). Vomiting, skin rash, and other side effects were equally seen in both groups but diarrhea was more frequent in the antibiotictreated group; $13(7 \%)$ in the intervention versus $5(2 \%)$ in the control group $(P<0.05)$.

\section{Discussion}

This study is a randomized controlled trial for the treatment of acute otitis media in children 6 months to 6 years of age. Two different therapeutic approaches are used in the management of AOM in children. According to the recent guidelines, the AOM recovery rate may not be much different in patients with or without antibiotic treatment. Therefore, both watchful waiting approach and antibiotic therapy are encouraged where appropriate [13].

According to our study, when antibiotic (high dose of amoxicillin) was prescribed to children with nonsevere AOM aged 6 months to 6 years, the AOM recovery rate was significantly higher compared to the watchful waiting group during the first 72 hours ( $73 \%$ vs. $44 \%$ ). In other studies, the AOM recovery in the antibiotic receiving groups ranged from $41 \%$ to $92.8 \%$ [18-21] versus $28 \%$ to $84 \%$ in the watchful waiting group [18-22]. These differences in the outcomes may be due to participants' age (younger or older than 2 years), type and different doses of antibiotics, i.e., amoxicillin 40 to $80 \mathrm{mg} / \mathrm{kg} / \mathrm{day}$, or amoxicillin-clavulanate, matching groups regarding vaccination against influenza, day care attendance, breastfeeding status, history of AOM recurrence, and exposure to smoke. Antimicrobial therapy for AOM has been attributed to be a major factor in the emergence of resistance among otopathogens; hence, current guidelines endorse withholding antibiotics as an 
TABLe 3: Association between treatment response and different factors.

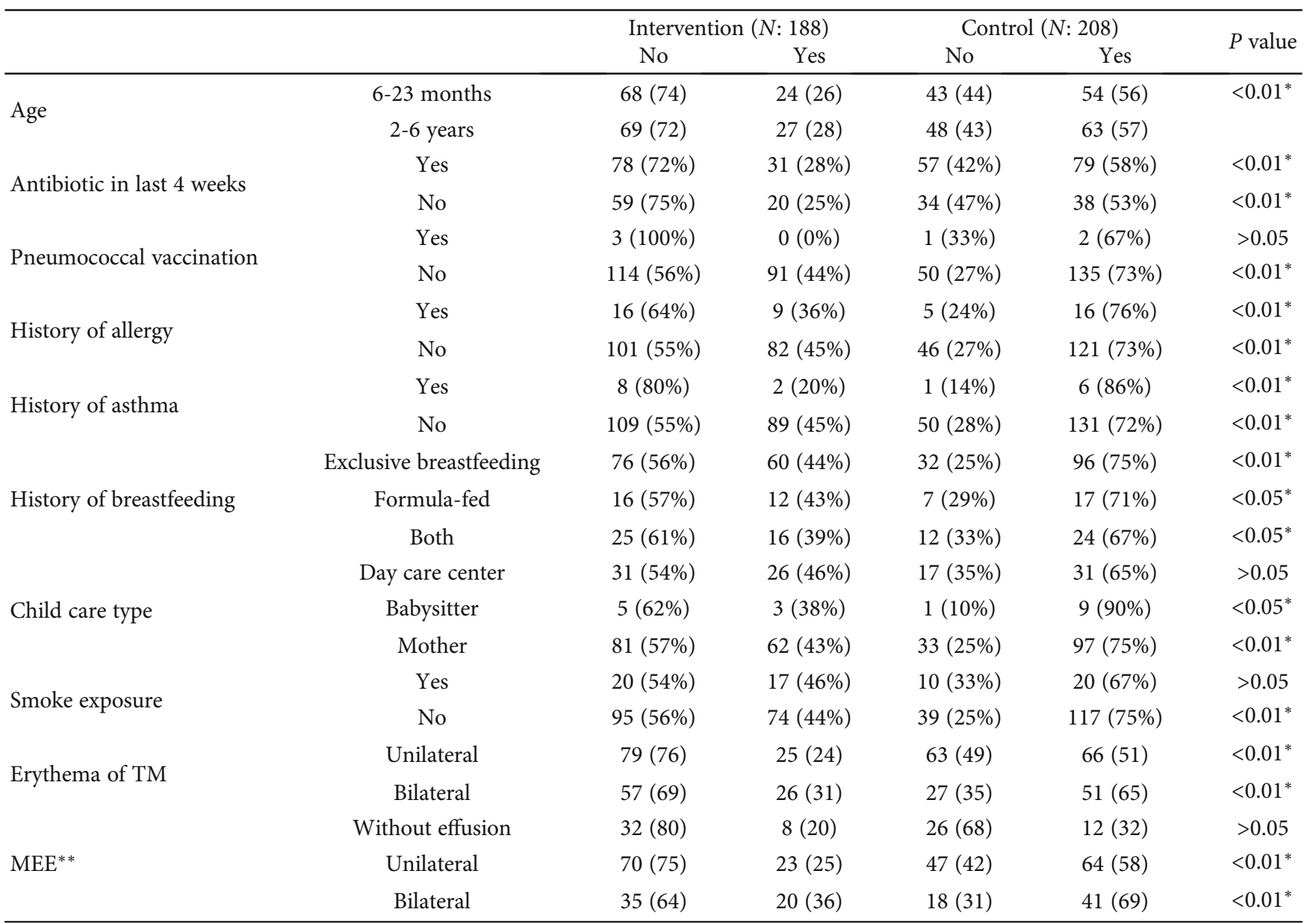

MEE: middle ear effusion; TM: tympanic membrane. ${ }^{*}$ Statistically significant. ${ }^{* *}$ All patients did not refer for examination.

TABLE 4: Follow-up findings in patients after the intervention.

\begin{tabular}{|c|c|c|c|c|}
\hline & Episode(s) & Intervention & Control & $P$ value \\
\hline \multirow{3}{*}{ AOM recurrence after 1 month } & 1 & $39(21 \%)$ & $50(24 \%)$ & \multirow{3}{*}{$>0.05$} \\
\hline & 2 & $16(8 \%)$ & $16(8 \%)$ & \\
\hline & $\geq 3$ & $6(3 \%)$ & $4(2 \%)$ & \\
\hline \multirow{3}{*}{ AOM recurrence between 1 and 3 months } & 1 & $0(0 \%)$ & $5(2 \%)$ & \multirow{3}{*}{$<0.05^{*}$} \\
\hline & 2 & $4(2 \%)$ & $14(7 \%)$ & \\
\hline & $\geq 3$ & $11(6 \%)$ & $9(4 \%)$ & \\
\hline MEE after 1 month & & $61(32 \%)$ & $70(34 \%)$ & $>0.05$ \\
\hline MEE after 3 months & & $15(8 \%)$ & $28(14 \%)$ & $>0.05$ \\
\hline Drug side effects & Total & $20(11 \%)$ & $6(3 \%)$ & $<0.01^{*}$ \\
\hline \multirow{3}{*}{ Type of side effects } & Diarrhea & $13(7 \%)$ & $5(2 \%)$ & $<0.05^{*}$ \\
\hline & Vomiting & $9(5 \%)$ & $4(2 \%)$ & $>0.05$ \\
\hline & Skin rash & $2(1 \%)$ & $0(0 \%)$ & $>0.05$ \\
\hline
\end{tabular}

MEE: middle ear effusion; AOM: acute otitis media. ${ }^{*}$ Statistically significant.

option in selected children where appropriate [18-23]. However, it should be considered that other studies have included children who were vaccinated against pneumococcal pathogens, which could have altered the AOM pattern in children. Studies on AOM have shown a $29 \%$ rate reduction of otitis media infections in children receiving this vaccine before the age of 24 months [24, 25]. Furthermore, a 40\% reduction of healthcare visits for otitis media has also been observed in children who were vaccinated $[26,27]$. Only $1.5 \%$ of children in our study were vaccinated with the pneumococcal- 
TABLE 5: Association between AOM recurrence after 3 months and different factors.

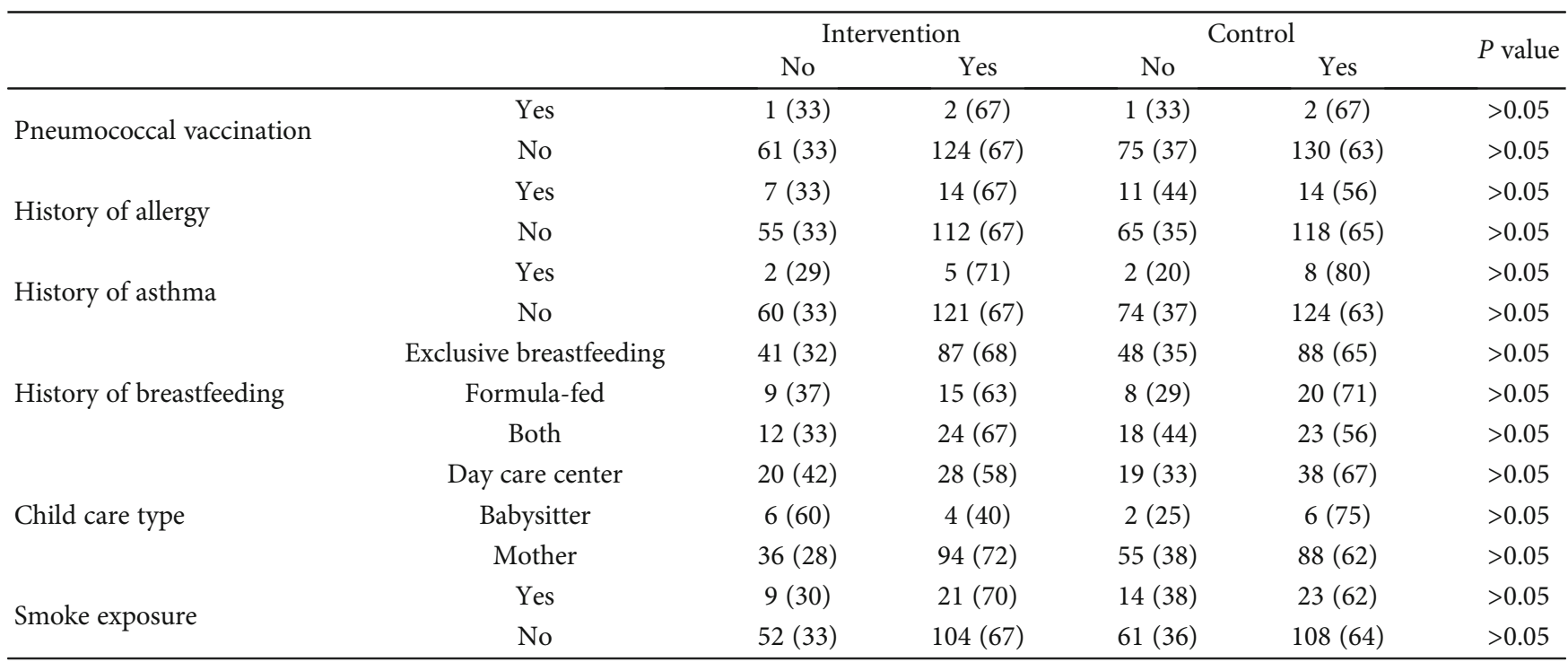

conjugated vaccine (PCV13). Therefore, due to the limited number of vaccinated participants, we could not evaluate the effect of this vaccine on AOM.

In our study, the use of antibiotics significantly improved the AOM in both age groups of 6 months to 2 years and 2 to 6 years old with or without the use of antibiotic treatment in the last 4 weeks prior to this study. Table 3 shows the other association between treatment response and different factors. However, the difference in improvement between the antibiotic vs. watchful waiting group was not observed in children that attended day care centers or exposed to smoke. Studies reported that exposure to smoke or attending day care centers are risk factors for AOM in children and may alter host defence against organism or exposed children to more virulent strains $[28,29]$. In addition, due to a limited number of patients who received pneumococcal vaccine, we could not have any discussions about the effect of these vaccines on AOM in our study.

In terms of adverse effects, our findings were in coordination with other studies, for example, diarrhea was more prevalent in the antibiotic-treated group, which was self-limited $[16-18,22]$. Nausea and vomiting were not significantly different in both groups. Despite the adverse effects of antibiotics, it seems that most of the patients may benefit from antibiotics and these mild side effects can be managed with proper follow-ups. Other side effects, which were reported previously like skin rash or eczema, were not seen in our patients [18]. In our study, no serious AOM complications such as meningitis, acute mastoiditis, or petrositis were observed in both groups but eardrum perforation was reported in two patients from the intervention group and one patient from the control group. Serious complications are rare in $\mathrm{AOM}$ patients, and eardrum perforation is the most common complication [30]. Therefore, due to the small sample size, we could not discuss the effect of antibiotics or watchful waiting approach on serious complications. No serious AOM-related adverse effects were reported in either groups of McCormick et al.'s study [22]. However, Hoberman et al. reported one case of acute mastoiditis in the placebo group. They have also reported tympanic perforation in $1 \%$ of the patients in the antibiotic group and 5\% in the placebo group [19]. Damoiseaux et al. and Tähtinen et al. reported tympanic perforation in $15.3 \%$ and $0.6 \%$ of the antibiotic group compared to $17 \%$ and $3.2 \%$ of the control group, respectively $[18,20]$.

Some of the long-term concerns regarding the management of AOM are the disease recurrence and persistence of MEE. It is not clear how antibiotic use or watchful waiting alters these consequences. In our study, no significant difference was found in the recurrence of AOM or persistence of MEE between two groups one-month postintervention. However, after 3 months, AOM recurrence was statistically higher in the control group. These findings were in coordination with some other studies $[18,22,24]$. Contrary to our findings, Hoberman et al.'s and Le Saux et al.'s studies reported that the recurrence rate of MEE and AOM were insignificantly higher in the control group after one month of treatment $[19,21]$. Based on these results, it is concluded that in either short-term (4-6 weeks) or long-term (3 months) follow-ups, the therapeutic choices may not alter the recurrence rate of AOM or MEE. We investigated factors such as the history of allergy or asthma, breastfeeding, child care type, and smoke exposure as effective factors in AOM recurrence or MEE in the two groups but we did not find any differences.

Our study showed that antibiotic therapy is superior in comparison to the observational approach in children who have not received pneumococcal vaccination. Antibiotic treatment is more beneficial in patients who can be monitored despite its adverse effects. The main limitation of our study was the lack of a placebo and not being able to perform a double-blinded study. Due to the infrequency of serious complications of AOM, further multicenter studies with numbered cases may be beneficial. Study on the effect of 
antibiotic usage in appearing more virulent strains and/or microbial resistance needs long-term studies and experienced laboratories.

\section{Conclusion}

In this study, we have investigated the appropriateness of the watchful waiting approach vs. antibiotic therapy in the management of AOM in a pediatric infectious diseases clinic of a tertiary hospital in Iran. It is concluded from our findings that despite the risk of bacterial resistance and potential side effects of antibiotics, faster symptom improvements are achieved when antibiotics are prescribed in children 6 months to 6 years with nonsevere AOM in our country. It could be attributed to the reason that pneumococcal vaccination is not included in the national vaccination program of Iran.

\section{Data Availability}

The trial data used to support the findings of this study are available from the corresponding author upon request.

\section{Ethical Approval}

The ethics committee of the Mazandaran University of Medical Sciences approved the study protocol (Code: IR.MAZUMS.REC.1395.2489), and it was registered in the Iranian registry of clinical trials (Code: IRCT20111224008507N2).

\section{Conflicts of Interest}

The authors declare that they have no conflicts of interest.

\section{Authors' Contributions}

All the authors did the conceptualization, methodology and investigation, analysis, and writing of the draft. MSR did the supervision.

\section{Acknowledgments}

We thank all patients and parents involved in management and treatment of AOM. The deputy of Research and technology of Mazandaran University of Medical Sciences provided the financial support of the study.

\section{References}

[1] J. Nokso-Koivisto, T. Marom, and T. Chonmaitree, "Importance of viruses in acute otitis media," Current Opinion in Pediatrics, vol. 27, no. 1, pp. 110-115, 2015.

[2] A. Vergison, R. Dagan, A. Arguedas et al., "Otitis media and its consequences: beyond the earache," The Lancet infectious diseases., vol. 10, no. 3, pp. 195-203, 2010.

[3] L. Monasta, L. Ronfani, F. Marchetti et al., "Burden of disease caused by otitis media: systematic review and global estimates," PLoS One, vol. 7, no. 4, article e36226, 2012.

[4] R. Kaur, M. Morris, and M. E. Pichichero, "Epidemiology of acute otitis media in the postpneumococcal conjugate vaccine era," Pediatrics, vol. 140, no. 3, article e20170181, 2017.
[5] R. C. Di Francesco, V. B. Barros, and R. Ramos, "Otite media com efusao em crianças menores de um ano," Revista Paulista de Pediatria (English Edition)., vol. 34, no. 2, pp. 148-153, 2016.

[6] N. Guys, T. Ramsey, A. Kandinov et al., "Interplay between socioeconomic status and otitis media disease burden in resource-rich and resource-poor nations," Otology \& Neurotology, vol. 39, no. 9, pp. e817-e824, 2018.

[7] C. Stockmann, K. Ampofo, A. L. Hersh et al., "Seasonality of acute otitis media and the role of respiratory viral activity in children," The Pediatric infectious disease journal., vol. 32, no. 4, pp. 314-319, 2013.

[8] G. Rahimzadeh, P. Gill, and M. S. Rezai, "Characterization of methicillin-resistant Staphylococcus aureus (MRSA) phages from sewage at a tertiary pediatric hospital," Archives of Pediatric Infectious Diseases, vol. 5, no. 1, 2016.

[9] G. Rahimzadeh, M. Saeedi, F. Farshidi, and M. S. Rezai, "Phage therapy in treatment of gram-negative bacterial infections: a systematic review," Journal of Mazandaran University of Medical Sciences, vol. 28, no. 165, pp. 203-212, 2018.

[10] T. Sakulchit and R. D. Goldman, "Antibiotic therapy for children with acute otitis media," Canadian Family Physician, vol. 63, no. 9, pp. 685-687, 2017.

[11] A. S. Lieberthal, A. E. Carroll, T. Chonmaitree et al., "The diagnosis and management of acute otitis media," Pediatrics, vol. 131, no. 3, pp. e964-e999, 2013.

[12] N. Le Saux, J. L. Robinson, C. P. Society, I. Diseases, and I. Committee, "Management of acute otitis media in children six months of age and older," Paediatrics \& Child Health, vol. 21, no. 1, pp. 39-44, 2016.

[13] S. Siddiq and J. Grainger, "The diagnosis and management of acute otitis media: American Academy of Pediatrics guidelines 2013," Archives of Disease in Childhood-Education and Practice., vol. 100, no. 4, pp. 193-197, 2015.

[14] H. G. Suzuki, J. E. Dewez, R. G. Nijman, and S. Yeung, "Clinical practice guidelines for acute otitis media in children: a systematic review and appraisal of European national guidelines," BMJ Open, vol. 10, no. 5, article e035343, 2020.

[15] M. Edmondson-Jones, T. Dibbern, M. Hultberg et al., "The effect of pneumococcal conjugate vaccines on otitis media from 2005 to 2013 in children aged $\leq 5$ years: a retrospective cohort study in two Swedish regions," Human Vaccines \& Immunotherapeutics, vol. 17, no. 2, pp. 517-526, 2021.

[16] A. C. Fortanier, R. P. Venekamp, C. W. Boonacker et al., "Pneumococcal conjugate vaccines for preventing acute otitis media in children," Cochrane Database of Systematic Reviews, vol. 5, 2019.

[17] J. L. Rubin, L. J. McGarry, D. R. Strutton et al., "Public health and economic impact of the 13-valent pneumococcal conjugate vaccine (PCV13) in the United States," Vaccine, vol. 28, no. 48 , pp. 7634-7643, 2010.

[18] R. A. Damoiseaux, F. A. van Balen, A. W. Hoes, T. J. Verheij, and R. A. de Melker, "Primary care based randomised, double blind trial of amoxicillin versus placebo for acute otitis media in children aged under 2 years," BMJ, vol. 320, no. 7231, pp. 350-354, 2000.

[19] A. Hoberman, J. L. Paradise, H. E. Rockette et al., "Treatment of acute otitis media in children under 2 years of age," The New England Journal of Medicine, vol. 364, no. 2, pp. 105-115, 2011.

[20] P. A. Tähtinen, M. K. Laine, P. Huovinen, J. Jalava, O. Ruuskanen, and A. Ruohola, "A placebo-controlled trial of 
antimicrobial treatment for acute otitis media," New England Journal of Medicine, vol. 364, no. 2, pp. 116-126, 2011.

[21] N. le Saux, I. Gaboury, M. Baird et al., "A randomized, doubleblind, placebo-controlled noninferiority trial of amoxicillin for clinically diagnosed acute otitis media in children 6 months to 5 years of age," CMAJ, vol. 172, no. 3, pp. 335-341, 2005.

[22] D. P. McCormick, T. Chonmaitree, C. Pittman et al., "Nonsevere acute otitis media: a clinical trial comparing outcomes of watchful waiting versus immediate antibiotic treatment," Pediatrics, vol. 115, no. 6, pp. 1455-1465, 2005.

[23] M. E. Pichichero, J. R. Casey, and A. Almudevar, "Reducing the frequency of acute otitis media by individualized care," The Pediatric infectious disease journal., vol. 32, no. 5, pp. 473-478, 2013.

[24] D. M. Spiro, K.-Y. Tay, D. H. Arnold, J. D. Dziura, M. D. Baker, and E. D. Shapiro, "Wait-and-see prescription for the treatment of acute otitis media: a randomized controlled trial," Journal of the American Medical Association, vol. 296, no. 10, pp. 1235-1241, 2006.

[25] K. A. Poehling, P. G. Szilagyi, C. G. Grijalva et al., "Reduction of frequent otitis media and pressure-equalizing tube insertions in children after introduction of pneumococcal conjugate vaccine," Pediatrics, vol. 119, no. 4, pp. 707-715, 2007.

[26] C. G. Grijalva, K. A. Poehling, J. P. Nuorti et al., "National impact of universal childhood immunization with pneumococcal conjugate vaccine on outpatient medical care visits in the United States," Pediatrics, vol. 118, no. 3, pp. 865-873, 2006.

[27] F. Zhou, A. Shefer, Y. Kong, and J. P. Nuorti, "Trends in acute otitis media-related health care utilization by privately insured young children in the United States, 1997-2004," Pediatrics, vol. 121, no. 2, pp. 253-260, 2008.

[28] S. Brophy-Williams, K. Jarosz, J. Sommer, A. Leach, and P. Morris, "Preventative and medical treatment of ear disease in remote or resource-constrained environments," The Journal of Laryngology \& Otology., vol. 133, no. 1, pp. 59-72, 2019.

[29] G. Aydemir and F. Ozkurt, "Otitis media with effusion in primary schools in Princes' Islands, Istanbul: prevalence and risk factors," The Journal of International Medical Research, vol. 39, no. 3, pp. 866-872, 2011.

[30] N. Principi, P. Marchisio, C. Rosazza, C. Sciarrabba, and S. Esposito, "Acute otitis media with spontaneous tympanic membrane perforation," European Journal of Clinical Microbiology \& Infectious Diseases, vol. 36, no. 1, pp. 11-18, 2017. 